\title{
A measuring equipment of no-load voltage ratio of operating distribution transformer based on harmonic spectrum technique
}

\author{
Qing-Long Xu, Yan-Zhen Wang, Fei Wang and Qiang Gao \\ Changshu Power Supply Company \\ Changshu, 215500, China \\ E-mail:xqlwin@163com
}

\begin{abstract}
The position of the tap switch is the main measure to adjust voltage, unfortunately, most of the position of tap switch was unknown because there were no information of the position on distribution transformer. This paper proposed a novel method to detect the position of the tap switch, the voltage and current of the transformer in both primary and secondary side were monitored, and then, the harmonic of the voltage and current were calculated. Finally, the position of the tap switch was determined by the measured voltage and current.
\end{abstract}

Keywords: Tap Switch; Voltage Measure; Current Measure; Harmonic.

\section{Introduction}

Power quality has been one of important aspects that companies are highly concerned. Voltage quality, one of power qualities, has attracted wide attentions from users in the past. Timely tap position shifting of distribution transformer in rural areas according to seasonal or load change is one of important guarantees of voltage qualified rate. In field operation, since the off-circuit tap-changer couldn't observe tap positions and there's no account system, the transformer is often discovered at the highest or the lowest tap position after power cut. Under this circumstance, it is impossible to regain qualified voltage at the low voltage side by adjusting tap position of the transformer, but has to resort other measures which will surely cause void power cut to users. With the increasing requirements on power distribution reliability, eradication of such void power cut must be enlisted into the agenda. Real-time detection of the actual operational tap position is an important basis to know whether voltage could be satisfied through tap position shifting of the transformer.

In traditional power distribution network, it is impossible to know the actual operational tap position of transformers due to the absent monitoring measures and complete voltage-current measurement data. Therefore, it is necessary to 
develop a set of portable no-load voltage ratio tester for distribution transformers, which could be used to make real-time test of no-voltage ratio of operating distribution transformers, determine actual operational tap position and prevent void power cut. Moreover, real-time measurement is conducive to recognize turn-to-turn short circuit in distribution transformers and grasp voltage distribution of $10 \mathrm{kV}$ circuit.

\section{Hardware Structure}

Hardware structure of this measuring equipment is shown in Figure 1.
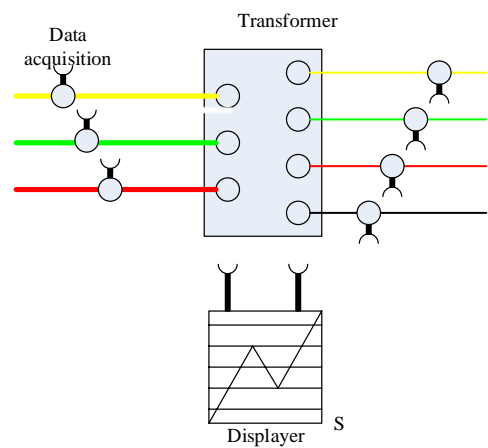

Fig. 1. System chart of the measuring equipment.

\subsection{Collection and wireless transmission of voltage and current at the high voltage side of distribution transformers}

The high voltage side is in the high field. There are two options available for voltage measurement: electromagnetic voltage changes or capacitive voltage changes. Electromagnetic voltage changes many have ferromagnetic resonance and the $10 \mathrm{kV}$-level capacitive voltage changes are seldom used in practical. This equipment made real-time voltage measurement by using earth coupling capacitance and made real-time current measurement by using iron core coupling capacitance. The iron core was closely related with accuracy of current measurement.

\subsection{Three-phase voltage and current data acquisition at low voltage side of distribution transformer}

Voltage at the low voltage side was $400 \mathrm{~V}$. Electric quantity could be measured directly through wire way or wireless way. When measuring voltages, voltage should be adjusted to the acceptable range of the equipment. 


\subsection{Portable terminal}

Main functions of portable control device are: (1) friendly human-computer interface; (2) receive electric quantity signal from high and low voltage sides with the wireless/wire communication module; (3) backstage data processing module for vector conversion of no-load voltage ratio.

Host unit is shown in Figure 2.

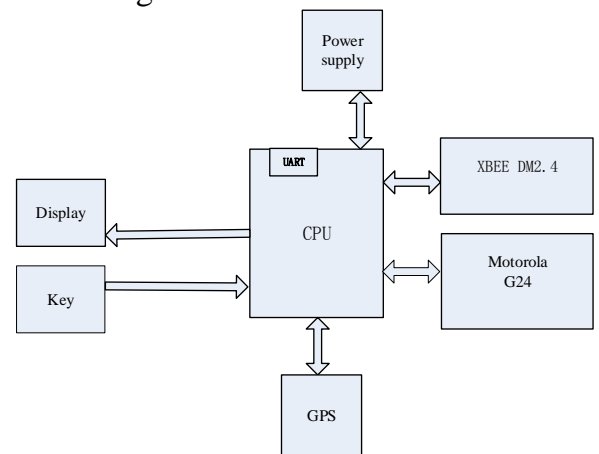

Fig. 2. Framework of portable terminal

\section{Main Software Algorithms}

No-load voltage test of this equipment mainly used the innovative algorithm which made a harmonic analysis on voltage and current signals at high and low voltage sides of the distribution transformer, and calculated the no-load voltage test under each harmonic.

Measured harmonic current at the low voltage side of distribution transformer is shown in Figure 3.

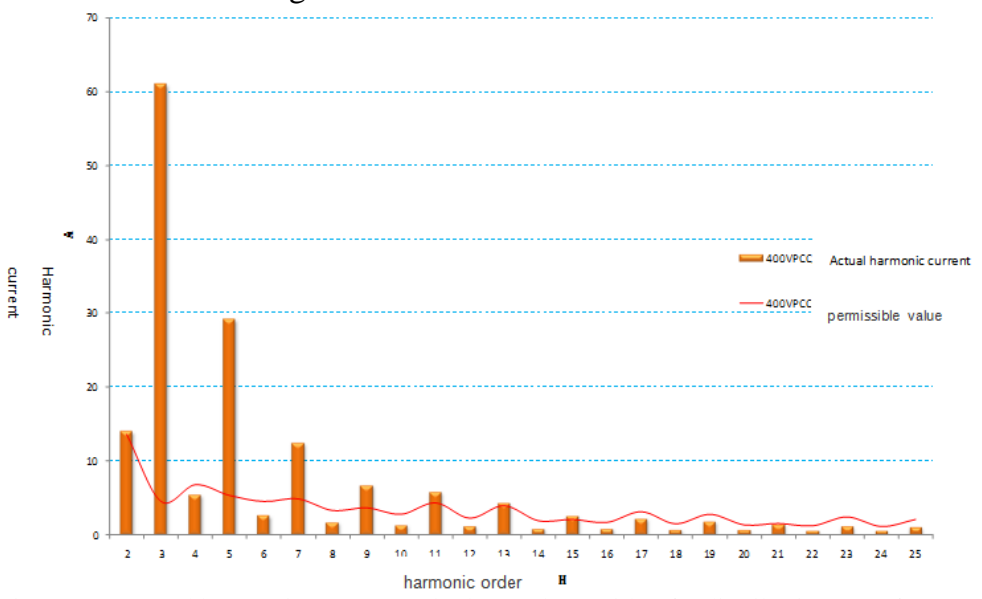

Fig. 3. Measured harmonic current at the low voltage side of a distribution transformer. 
No-load voltage calculation considered voltage and current. Voltage was calculated through weighting of voltage ratio between fundamental wave and each harmonic at the high and low voltage sides. Current was calculated through weighting of current ratio between fundamental wave and each harmonic at the high and low voltage sides. Weight of related voltage components was generally higher than that of related current components: weight of voltage components and weight of current components were equal (50\%) when the distribution transformer was under rated load. When the distribution transformer was under empty load, no-load voltage was calculated from voltage component and weight of current component was 0 . When load of the distribution transformer decreased from full load to empty load, weight of voltage component increased linearly from $50 \%$ to $100 \%$, while weight of current component decreased linearly from $50 \%$ to 0 . Finally, current no-voltage voltage of the distribution transformer was calculated through overall weighted average and the actual tap position was displayed. The calculation formula is:

$$
\begin{gathered}
k_{U}=\sum m_{i}\left[\left(\frac{U_{i}^{\text {high_l1 }}}{U_{i}^{\text {low } l 1}}+\frac{U_{i}^{\text {high_l2 }}}{U_{i}^{\text {low } l 2}}+\frac{U_{i}^{\text {high } l s}}{U_{i}^{\text {low_ls }}}\right) / 3\right] \\
k_{I}=\sum n_{i}\left[\left(\frac{I_{i}^{\text {low } w_{-} l 1}}{I_{i}^{\text {high_l1 }}}+\frac{I_{i}^{\text {low } l 2}}{I_{i}^{\text {high } l 2}}+\frac{I_{i}^{\text {low } l s}}{I_{i}^{\text {high_ls }}}\right) / 3\right] \\
k=\alpha k_{U}+(1-\alpha) k_{I} \\
\sum m_{i}=1, \sum n_{i}=1, \alpha=0.5 \times \frac{s}{s_{B}}
\end{gathered}
$$

where $k_{U}$ is no-load voltage of voltage component, $k_{I}$ is no-voltage

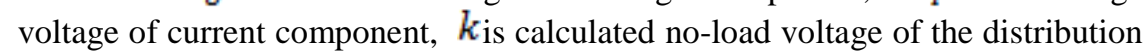
transformer, $m_{i}$ is weight of voltage harmonics, $n_{i}$ is weight of current harmonics, $\alpha_{\text {is }}$ weight harmonics of $k_{U}, S$ is actual load of the distribution transformer, $S_{B}$ is rated load of the distribution transformer. $U_{i}^{\text {high }}{ }^{l 1}$ is the $i^{\text {th }}$ voltage harmonic on coil 1 at the high voltage side, $U_{i}^{\text {low }}{ }^{l 1}{ }^{i}$ is the $i^{\text {th }}$ voltage harmonic on coil 1 at the low voltage side $I_{i}^{l o w_{-} l 1}$ is the $i^{\text {th }}$ current harmonic on coil 1 at the low voltage side, and $I_{i}^{\text {high } l 1}$ is the $i^{\text {th }}$ current harmonic on coil 1 at the high voltage side.

It can be seen from Eq. (1) that was the voltage ratio between the high voltage side and the low voltage side, and was the current ratio between the high voltage side and the low voltage side. No-load voltage ratios of different harmonics were calculated from voltage and current at different coils. The noload voltage ratio of the ith harmonic was the average of calculated no-load voltages of three coils. Therefore, collected voltage and current values at the 
high and low voltage sides must be converted into different coils in practical calculation.

\section{Interface Implementation}

Tap position of the distribution transformer was displayed by the monitor terminal and mobile phone together. The monitor terminal was responsible for collecting real-time waveform and FFT to get average voltage, average current, fundamental wave value and values of harmonics. Data were uploaded to the server regularly. The final tap position was displayed by mobile phone. Based on the client interface, terminal number installed at the high and low voltage sides was connected to the distribution transformer by wires to update data and acquire the desired latest voltage and current data from the high and low voltage sides. Next, it will calculate the no-load voltage ratio according to Eq. (1). Android smart phone was used as the client of interface display, which was convenient for development, installation and use.

The data communication structure is shown in Figure 4.

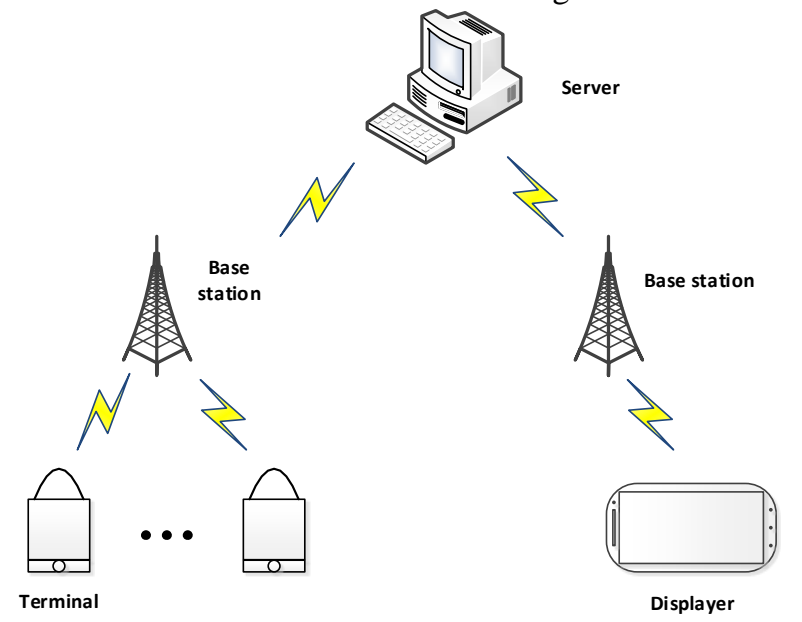

Fig. 4. Data communication structure.

According to the overall structure and actual computation demand, the client end shall be equipped with terminal selection as well as selection and updating of connection mode of the distribution transformer except tap position display. Moreover, related voltage, current, updating time and other related information were displayed simultaneously. The display interface is shown in Figure 5. 


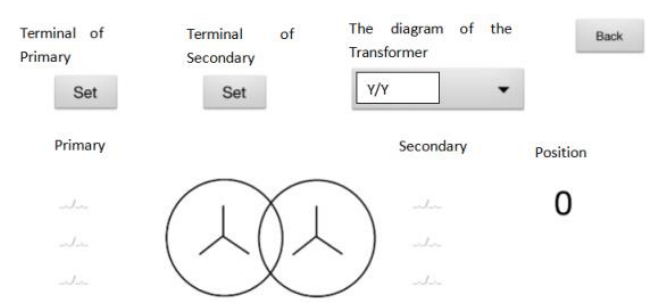

Fig. 5. Tap position display interface design

It can be seen from Figure 5 that the upper interface was terminal selection and connection mode selection, whereas the lower interface was the sketch map of transformer. Effective voltage and current values at the high and low voltage sides were displayed at the left and right of the lower sketch map of transformer. The right side was tap position display and data updating buttons. A total of three tap positions were optional: $5 \%, 0 \%$ and $-5 \%$. The bottom interface showed uploading time of the latest data of two terminals.

After terminals at the high and low voltage sides of the distribution transformer were chosen, terminal number would be displayed at the corresponding positions. The sketch map of the distribution transformer would change once the connection mode was changed. Finally, click the updating button and calculate the no-load voltage ratio. This no-load voltage ratio would be compared with the standard no-load voltage ratio of the distribution transformer, getting the tap position data and updating display accordingly.

\section{Field Test}

This equipment was tested on a 10/0.4 transformer on April 19th, 2016. The transformer chose the angle-star connection mode. The No.10 monitor terminal was installed at the high voltage side and the No.23 monitor terminal was at the low voltage side. The actual installation is presented in Figure 6.

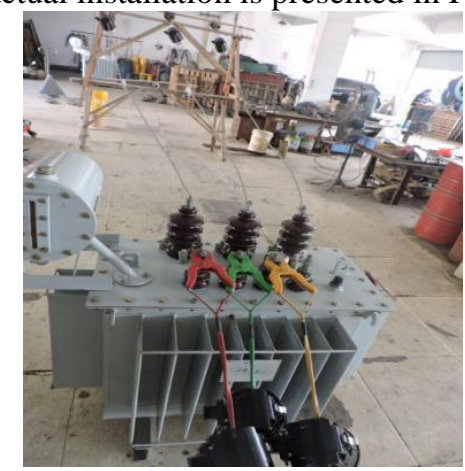

Fig. 6. Field installation of monitor terminals. 
Three tests were conducted. The secondary terminal voltage was maintained $400 \mathrm{~V}$, while the first terminal voltage was $10.5 \mathrm{kV}, 10 \mathrm{kV}$ and $9.5 \mathrm{kV}$, corresponding to 1,2 and 3 tap positions. The display interface showed the connection mode and tap position data of the distribution transformer accurately, indicating that the proposed algorithm was feasible and effective. The whole system has been operated successfully and displayed tap position of the distribution transformer accurately.

\section{Conclusions}

The transformer has been analyzed and researched concretely in this paper. The author designed the modular hardware structure and compiled the software algorithm based on harmonic spectrum program. Through the actual device test shows the correctness of this topic.

\section{References}

1. Qinglong Xu, Development of Portable Digital HVDC Genetator Based on $M C U$ and CPLD, (APPEEC'12),(Shanghai, China, 2012).

2. XU Qing-long and ZHOU Wen-hua, Analysis and Control of Quality Factor $\mathrm{Q}$ in Series Resonance Voltage Endurance Test, Journal.(2011)

3. XU Qing-long and $\mathrm{Wu}$ wei, Analysis of Two Fault Cases Happened in the Arc-Suppression Coil System, Jiangsu Electrical Engineering.(2012)

4. Su Ning, Development of combined Monitoring System of MOA insulating material and CT insulating material, (MAEE'14),(Nanjing, China, 2014).

5. Wenyu Kong, Grounding current monitoring system for transformer core material , (MAEE'15),(Beijing, China, 2015). 